\title{
Grids of model spectra for WN stars, ready for use
}

\author{
W.-R. Hamann and G. Gräfener \\ Lehrstuhl Astrophysik der Universität Potsdam, Am Neuen Palais 10, 14469 Potsdam, Germany \\ e-mail: wrh@astro.physik.uni-potsdam. de \\ Received 24 March 2004 / Accepted 20 July 2004

\begin{abstract}
Grids of model atmospheres for Wolf-Rayet stars of the nitrogen sequence (WN subclass) are presented. The calculations account for the expansion of the atmosphere, non-LTE, clumping, and line blanketing from iron-group elements. Observed the presented model grids cover the whole relevant range of stellar temperatures and mass-loss rates. We point out that there is a degeneracy of parameters for very thick winds; their spectra tend to depend only on the ratio $L / \dot{M}^{4 / 3}$. Abundances of the calculated grids are for Galactic WN stars without hydrogen and with $20 \%$ hydrogen (by mass), respectively. Model spectra and fluxes are available via internet (http://www. astro.physik. uni-potsdam.de/PoWR.html).
\end{abstract} \\ spectra of single Galactic WN stars can in general be reproduced consistently by this generation of models. The parameters of
}

Key words. stars: mass-loss - stars: Wolf-Rayet - stars: atmospheres

\section{Introduction}

Massive stars are important drivers of the cosmic evolution. Current interest concerns their role in the early universe, and as progenitors of supernovae and $\gamma$-ray bursts. Nevertheless, the evolution of massive stars is not yet safely established, and their physics is not well understood. Much of the problem is related to the mass-loss phenomenon, which is especially strong in Wolf-Rayet stars.

Strong efforts were made in the last two decades to establish the parameters of Wolf-Rayet stars empirically. The main problem is the calculation of adequate model atmospheres which are prerequisite for a quantitative spectral analysis. Such models have to account for extreme non-LTE situations, complex model atoms, and the supersonic expansion of the atmosphere. Corresponding codes have been developed independently by Hillier $(1987 a, b)$ and by our group (Hamann \& Schmutz 1987). First generations of models accounted only for helium and hydrogen when they were employed for a comprehensive analysis of the Galactic WN stars (Hamann et al. 1995). The next step was the inclusion of the CNO elements. A corresponding study of Galactic WN stars was provided by Hamann \& Koesterke (1998a, hereafter Paper I). On the same level of sophistication, WN stars in the LMC (Hamann \& Koesterke 2000), and WC stars in the Galaxy (Koesterke \& Hamann 1995) and in the LMC (Gräfener et al. 1998) have been analyzed.

The major deficiency of this generation of model atmospheres was the neglect of line blanketing by the millions of lines from iron and iron-group elements. Hillier \& Miller (1998) were the first to include this important effect into their code. Their models have been applied for the analysis of various stars (e.g., Hillier \& Miller 1999; Crowther et al. 2000, 2002; Dessart et al. 2000; Herald et al. 2001). The lineblanketed version of the Potsdam code became available with Gräfener et al. (2002).

The best way to analyze a larger sample of stars systematically is first to establish "models grids", i.e. sets of model atmospheres and synthetic spectra which cover the relevant range of parameters. Grids of unblanketed models for WN stars were presented in Paper I. The present paper is the first to publish grids of models from the new, iron line blanketed generation. Internet access to the synthetic spectra is provided to the community.

The rest of the paper is organized as follows. In Sect. 2 our model calculations are characterized. The obtained model grids are described in Sect. 3. In Sect. 4 we draw the attention to a degeneracy in the parameter space for very dense winds. The web interface giving access to the model grids is introduced in Sect. 5. The paper is concluded with an outlook in Sect. 6.

\section{The models}

\subsection{Basic assumptions}

The models are based on the "standard" assumptions for Wolf-Rayet stars. The atmosphere expands in a spherically symmetric, stationary flow. With a given mass-loss rate $\dot{M}$, the density stratification and the velocity field $v(r)$ are related via the equation of continuity.

The velocity field is pre-specified in the common way. For the supersonic part we adopt the usual $\beta$-law, with the terminal velocity $v_{\infty}$ being a free parameter. The exponent $\beta$ is set to unity throughout this work. In the subsonic region the velocity 
field is defined such that a hydrostatic density stratification is approached (Hamann \& Schmutz 1987).

The "stellar radius" $R_{*}$, which is the inner boundary of our model atmosphere, corresponds by definition to a Rosseland optical depth of 20. The "stellar temperature" $T_{*}$ is defined by the luminosity $L$ and the stellar radius $R_{*}$ via the StefanBoltzmann law, i.e. $T_{*}$ denotes the effective temperature referring to the radius $R_{*}$.

In contrast to our model grids published previously, wind inhomogeneities ("clumping") are now accounted for. In a firstorder approximation (cf. Hamann \& Koesterke 1998b), we assume that optically thin clumps fill a volume fraction $f_{\mathrm{V}}=D^{-1}$ while the interclump space is void. Thus the matter density in the clumps is higher by a factor $D$, compared to an un-clumped model of same parameters.

\subsection{Model calculations}

The presented models are calculated with the Potsdam code for expanding stellar atmospheres. Non-LTE opacities and emissivities are evaluated for complex model atoms (see Table 1 for a summary of the applied model atoms). Only Doppler broadening is accounted for in the profile function of the line absorption coefficient. The Doppler velocity $v_{\mathrm{D}}$ reflects random motions on small scales and is set to $100 \mathrm{~km} \mathrm{~s}^{-1}$. A "microturbulence" of that order-of-magnitude reproduces best the detailed shape of the observed line profiles.

The radiation transfer is formulated in the co-moving frame of reference (CMF). The moment equations are solved by a differencing scheme (see Gräfener et al. 2002, for more details). The variable Eddington factors are updated by solving the angle-dependent radiation transfer with a short-characteristics integration (Koesterke et al. 2002).

The statistical equations balance all relevant radiative and collisional transition rates. The consistent solution of radiative transfer and statistical equilibrium is achieved by means of an Accelerated Lambda Iteration (ALI) technique (e.g. Hamann 2003).

The temperature stratification is obtained from the constraint of radiative equilibrium. It is established by a temperature correction scheme generalized from the ideas of Unsöld and Lucy, and accelerated by an ALI technique as well (Hamann \& Gräfener 2003). This scheme achieves a very accurate solution of the energy equation. The improvement of our new models compared to our previously published grids is partly due to the superior temperature stratification.

The overall convergence of the scheme is stable but not very fast, and requires typically hundreds of cycles, mainly to establish the temperature structure with the desired accuracy. The computing time per model amounts to about one day on a modern workstation.

After the non-LTE population numbers have been obtained, the atomic data are further refined by splitting the levels and multiplets in the observed spectral range as far as necessary, and the emergent spectra are calculated in the observer's frame ("formal integral"). Frequency redistribution by electron scattering is taken into account in this step.
Table 1. Model atom summary. The entries for Fe refer actually to superlevels and superlines of a generic element representing the whole iron group from $\mathrm{Sc}$ to $\mathrm{Ni}$.

\begin{tabular}{lrr}
\hline \hline Ion & No. of levels & No. of lines \\
\hline He I & 17 & 55 \\
He II & 16 & 120 \\
He III & 1 & 0 \\
N II & 1 & 0 \\
N III & 33 & 118 \\
N IV & 38 & 153 \\
N v & 17 & 80 \\
N VI & 1 & 0 \\
C II & 3 & 2 \\
C III & 40 & 224 \\
C IV & 21 & 140 \\
C V & 1 & 0 \\
Fe III & 1 & 0 \\
Fe IV & 11 & 49 \\
Fe V & 13 & 69 \\
Fe VI & 17 & 121 \\
Fe VII & 11 & 52 \\
Fe VIII & 9 & 34 \\
Fe IX & 9 & 35 \\
Fe X & 1 & 0 \\
\hline 20 ions & 261 & 1252 \\
\hline
\end{tabular}

The major improvement of our WR model atmospheres, compared to Paper I, concerns the inclusion of iron line blanketing. The problem of treating iron and the other iron group elements (nickel etc.) in atmosphere models comes from the enormous complexity of their electron system. Present data collections comprise $\approx 10^{5}$ energy levels and $\approx 10^{7}$ line transitions between those levels.

A full non-LTE treatment of such model atoms is not currently feasible, but the "superlevel" approach allows an approximate treatment. Its basic idea is to group the numerous energy levels into a much smaller number (say, per ion 10-20) of "superlevels" for which non-LTE population numbers are calculated. Within each superlevel, the relative population of the individual levels is assumed to be in LTE.

The radiative transition between two superlevels forms a "superline". Each superline is composed of all individual lines between the individual levels of the involved superlevels. Therefore the profile function covers a broad band with a complicated shape.

In static atmospheres there are techniques to simplify the radiative transfer for those complicated superline profiles. For instance, the frequencies might be re-arranged according to the opacity and then represented by an "opacity distribution function (ODF). This cannot be applied in moving atmospheres, because each frequency point is coupled to its neighbors by Doppler shifts and therefore not relocatable. The only way out is to treat all these complicated superline profiles as they are, and resolve the individual lines with a sufficiently fine spacing of frequencies (typically $10^{5}$ frequency points).

Details of our line-blanketing formalism are given in Gräfener et al. (2002). As described there (Table 2), a generic 
ion is constructed from the atomic data of the iron-group elements from $\mathrm{Sc}$ to $\mathrm{Ni}$, assuming relative abundances as in the sun. When grouping the energy levels into superlevels, we take care that no energy band is wider than $5 \times 10^{4}$ Kayser (wavenumber in $\mathrm{cm}^{-1}$ ), corresponding to $2000 \AA$. The resulting numbers of superlevels and of the transitions between them are included in Table 1. A limitation of the present iron model atom is the neglect of ions higher than Fe IX. Future generations of our models will also account for the so-called M-shell ions Fe IX to Fe XVI. While the full set of iron-group line data from Kurucz (1991) is used for calculating population numbers and temperature stratification, only the subset with experimentally confirmed wavelengths is applied when the formal integral is evaluated for the synthetic spectrum.

In many WR stars the iron lines form a dense "forest" in the UV. Figure 1 shows two examples. WR 1 is of early subtype; numerous lines from Fe V and Fe VI are crowding shortward of $1500 \AA$ in the spectrum of this very hot $\left(T_{*}=\right.$ $141 \mathrm{kK}$ ) star. WR 40 has a late subtype (WN8) and is much cooler $\left(T_{*}=45 \mathrm{kK}\right)$; in this spectrum an "iron forest" appears between 1500 and $2000 \AA$ and is due to Fe IV. (The latter star has been modeled in detail by Herald et al. 2001.) Generally the pseudo-continuum formed by the crowding of iron lines is well reproduced, with only a few individual features not being matched. Thus our line-blanketing formalism works satisfactory.

Iron line blanketing has a significant effect on the resulting models. The main effect is a steeper drop of temperature and ionization with increasing radial coordinate. Therefore, lines from lower ions (such as He I) can form in the outer layers of an atmosphere together with lines from high ions (as $\mathrm{N} \mathrm{V}$ ) from deep layers. This leads to a much more consistent reproduction of the observed spectra, as will be demonstrated in the next section.

Complex model ions of H, He, N III-N V and C III-C IV are accounted for in our models. Due to the neglect of N II, the corresponding lines which might be detectable in the coolest WNL stars are not included in our synthetic spectra. Further trace elements, such as $\mathrm{O}, \mathrm{Ne}, \mathrm{Si}, \mathrm{S}, \mathrm{Ar}$ and $\mathrm{Ca}$, are not included in our present model generation. Potentially this might cause deviations from models with more complete atomic data, especially in the far-UV flux. Comparisons have been made between WC models from our code and from Hillier's code with inclusion of these trace elements, and revealed good consistency, including ionizing fluxes (Crowther 2003).

A still questionable point is the treatment of the dielectronic recombination to N III, N IV and C III. In our previous papers we employed the approximation proposed by Mihalas \& Hummer (1973). For the doubly excited states close to the ionization threshold, LTE population with respect to the next-higher ion was assumed, and the lines from and to those levels ("stabilizing transitions") were treated in the optically thin approximation because they are very broad due to the short life time of the autoionization levels. The effect of this dielectronic recombination treatment, namely a strengthening of lines from low ions (N III), was highly welcome. However, iron line-blanketing, via a steeper temperature gradient now achieves a similar effect. The additional action of our
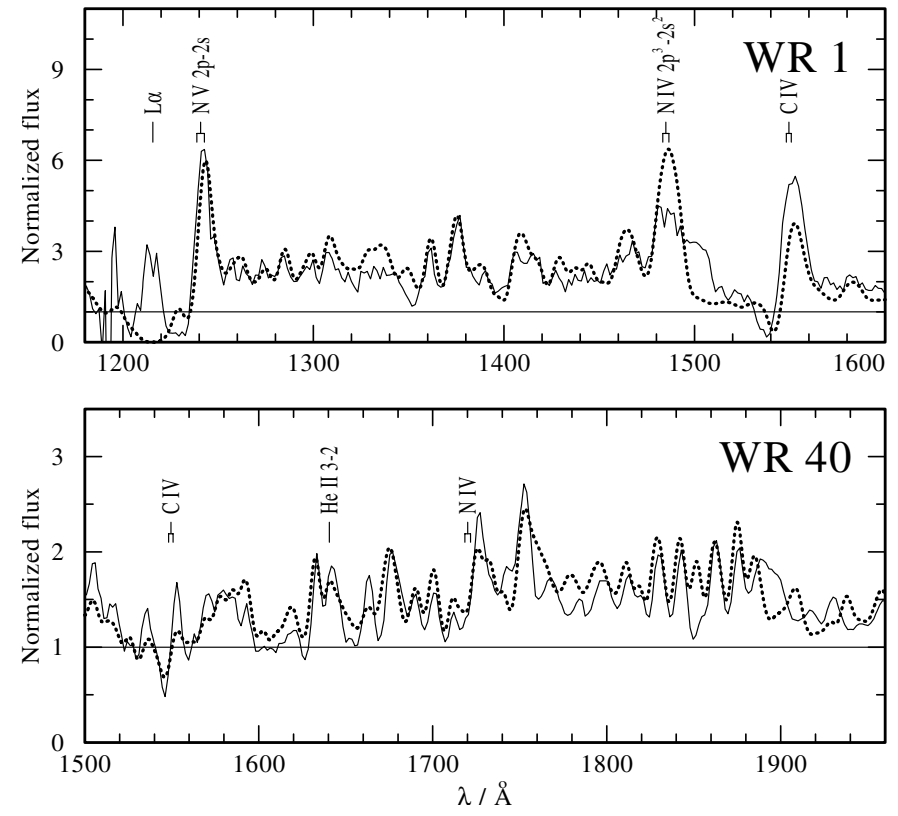

Fig. 1. Forest of iron lines in the UV. Models from our grid (dotted lines) are compared with low-resolution IUE observations (full lines). Upper panel: WR 1 (WN5-s), WNE model 16-20 with $T_{*}=141 \mathrm{kK}$ and $\log R_{\mathrm{t}} / R_{\odot}=0.1$; an "iron forest" appears shortward of $1500 \AA$ in the spectrum of this very hot star and is mainly due to Fe $\mathrm{V}$ and $\mathrm{Fe}$ VI. Lower panel: WR 40 (WN8), WNL model 06-14 with $T_{*}=45 \mathrm{kK}$ and $\log R_{\mathrm{t}} / R_{\odot}=0.7$; in this spectrum of late subtype an "iron forest" appears between 1500 and $2000 \AA$ and is due to Fe IV. Generally the pseudo-continuum formed by the crowding of iron lines is well reproduced by the models, with only a few individual features not being matched.

dielectronic-recombination treatment leads to spurious spectral features and spoils the agreement between synthetic and observed spectra. We made experiments (with C III and WC stars) with an alternative treatment of the dielectronic recombination in the form of "resonances" in the photoionization cross section, and found that the effect is actually quite small. It seems that the Mihalas \& Hummer (1973) assumptions, in the first place probably the optically thin treatment of the stabilizing transitions, lead to a severe overestimation of the recombination rates. Therefore we decided to leave out the dielectronic recombination channel completely for our present model grids.

\section{Grids of WN models}

If we adopt the "standard" assumptions described in Sect. 2.1, any particular WR model atmosphere is specified by its chemical composition and the parameters $L, T_{*}, \dot{M}, v_{\infty}$ and $D$. For the purpose of calculating grids of models we must restrict this parameter space.

The chemical abundances of WN stars are dominated by helium. The CNO process has converted most of the initial C, $\mathrm{N}$ plus $\mathrm{O}$ content into nitrogen. An initially solar mixture thus yields a nitrogen abundance of about $1.5 \%$ (by mass). Spectral analyses confirmed that this value is appropriate for Galactic WN stars, while carbon is depleted to about $10^{-4}$. Some peculiar WN stars, with spectral classifications WN/WC, have considerably stronger traces of carbon. For the iron-group 
abundance we adopt a mass fraction of $1.4 \times 10^{-3}$, slightly above the solar value. This value is plausible for Galactic Population I stars, and reproduces the observed "iron forest" well (cf. Fig. 1). Within the iron group, the relative abundances are assumed to be solar (see Gräfener et al. 2002, Table 2).

It was a major discovery that late-type WN (short: WNL) stars display some residual hydrogen (Conti et al. 1983; Hamann et al. 1991, 1995; Paper I, while most early-type WN (short: WNE) atmospheres are apparently hydrogen-free. Therefore we present two model grids with different chemical composition. One grid, termed "WNE" for brevity, is hydrogen-free, while the models of a second grid named "WNL" contain some hydrogen. The observed $\mathrm{H}$ abundances cover a wide range; for our WNL grid we adopt $20 \%$ (by mass) hydrogen as a roughly typical number.

WNE stars have faster winds than WNL stars (see Hamann et al. 1995, Fig. 3). In order to choose representative values, we set $v_{\infty}$ to $1600 \mathrm{~km} \mathrm{~s}^{-1}$ for the WNE and to $1000 \mathrm{~km} \mathrm{~s}^{-1}$ for the WNL grid, respectively. Further grids with different $v_{\infty}$ will be added to the data base in the future.

With the described choices, the parameter space of each grid is spanned by the parameters $L, T_{*}$ and $\dot{M}$. Fortunately it can be reduced by one dimension with the help of the scaling law for WR spectra found by Schmutz et al. (1989). With inclusion of the density contrast $D$ (Hamann \& Koesterke 1998b), we define the "transformed radius" $R_{\mathrm{t}}$ by

$R_{\mathrm{t}}=R_{*}\left[\frac{v_{\infty}}{2500 \mathrm{~km} \mathrm{~s}^{-1}} \mid \frac{\dot{M} \sqrt{D}}{10^{-4} M_{\odot} \mathrm{yr}^{-1}}\right]^{2 / 3}$

Models of same $R_{\mathrm{t}}$ show very similar spectra, irrespective of the individual combination of the parameters entering Eq. (1). While profile shapes vary with $v_{\infty}$, the equivalent widths of the emission lines depend in first approximation only on $R_{\mathrm{t}}$. When fitting observed spectra, one should therefore aim at reproducing the equivalent width in those cases where the profile shape cannot be matched due to inadequate $v_{\infty}$ of the available models. All absolute fluxes scale proportional to $R_{*}^{2}$.

Therefore we can restrict our model grids to a pre-selected stellar luminosity, for which we take $\log L / L_{\odot}=5.3$ as a typical value. Then only two parameters, $T_{*}$ and $R_{\mathrm{t}}$, have to be varied. The resulting models can be easily applied to stars of different luminosity with the same set of $T_{*}$ and $R_{\mathrm{t}}$, just by scaling the absolute fluxes to the different $L$. Equation (1) implies that for fixed $R_{\mathrm{t}}$ the mass-loss rate $\dot{M}$ scales proportional to $L^{3 / 4}$.

The clumping contrast $D$ has only little influence on the spectra, but the mass-loss rate obtained from a spectral fit scales with $D^{-1 / 2}$. For the model grids we adopt $D=4$ as a typical value established for WN stars (Hamann \& Koesterke 1998b).

The fixed grid parameters are compiled in Table 2, while $T_{*}$ and $R_{\mathrm{t}}$ are variable. For convenience we introduce an indexing scheme of the grid models. Both $T_{*}$ and $R_{\mathrm{t}}$ are varied in logarithmic steps. We compose a model designation in the form $k-m$ from two two-digit indices $k$ and $m$ (for instance, 09-13), where $k$ defines the stellar temperature

$\log \left(T_{*} / \mathrm{K}\right)=4.35+0.05 k$
Table 2. Model grid parameters. Only the helium and hydrogen abundances, and the terminal wind velocity are different for the grids termed "WNE" and "WNL", respectively. Abundances are given as mass fractions.

\begin{tabular}{lcc}
\hline \hline & WNE grid & WNL grid \\
\hline$X_{\mathrm{H}}$ & 0.00 & 0.20 \\
$X_{\mathrm{He}}$ & 0.98 & 0.78 \\
$X_{\mathrm{C}}$ & \multicolumn{2}{c}{$1.0 \times 10^{-4}$} \\
$X_{\mathrm{N}}$ & \multicolumn{2}{c}{$1.5 \times 10^{-2}$} \\
$X_{\mathrm{Fe}}$ & \multicolumn{2}{c}{$1.4 \times 10^{-3}$} \\
$\log L / L_{\odot}$ & \multicolumn{2}{c}{5.3} \\
$v_{\infty} / \mathrm{km} \mathrm{s}^{-1}$ & 1600 & 1000 \\
$D$ & & 4 \\
\hline
\end{tabular}

and $m$ codes the transformed radius

$\log \left(R_{\mathrm{t}} / R_{\odot}\right)=2.1-0.1 \mathrm{~m}$.

As all models within a grid have the same luminosity, the stellar radius $R_{*}$ scales proportional to $T_{*}^{-2}$ according to StefanBoltzmann's law. Thus Eq. (1) implies that the mass-loss rate scales with the grid parameters as $\dot{M}^{2 / 3} \propto T_{*}^{-2} R_{\mathrm{t}}^{-1}$. With the chosen spacing of our grid points, the mass-loss rate depends just on the difference $k-m$,

$\log \left(\frac{\dot{M}}{M_{\odot} \mathrm{yr}^{-1}}\right)=m_{0}-0.15(k-m)$

In other words, when moving one grid step towards higher $T_{*}$ and one grid step towards smaller $R_{\mathrm{t}}$, the mass-loss rate is not changed. For our choice of $D$ and $v_{\infty}$, the constant $m_{0}$ is -5.434 and -5.638 for the WNE and the WNL grid, respectively.

In our calculated WNE grid the stellar temperature varies between 31.6 and $188 \mathrm{kK}(k=3, \ldots, 18)$, and the transformed radius lies between 1 and 50. The mass-loss rates of the models range between $\log \left[\dot{M} /\left(M_{\odot} \mathrm{yr}^{-1}\right)\right]=-6.184$ and -3.784 for the grid's standard luminosity. These parameters cover the whole range where WNE stars are found. For the WNL grid we omitted stellar temperatures higher than $100 \mathrm{kK}$ because stars with hydrogen are usually not that hot. Instead, we added models with cooler temperatures $(28.3 \mathrm{kK})$ to that grid.

As already announced in the previous section, we will now demonstrate that the line-blanketed models allow a much more consistent reproduction of the observed spectra than the previous, unblanketed models. Figure 2 shows the observed spectrum of WR 44, an early-type WN star with weak lines (WN4-w). It is compared with a WNE grid model (09-12) which is a good fit to the helium lines, including He I 5876. Note that this model reproduces also the $\mathrm{N} v$ lines, namely the N v 4933-4944 multiplet and - not perfectly, but within a factor of two - the doublet N v 4603,4619. The reader might compare this fit with the analogous plot in Paper I, Fig. 5. There is a dramatic progress, as the unblanketed models used in Paper I never showed He I and N V simultaneously.

In Paper I we fortunately trusted more in the $\mathrm{N} V$ than in the He I lines when determining the best-fit parameters. In the shown case of WR 44 we had adopted model 10-11 as the final fit, although He I 5876 was completely absent in that model 


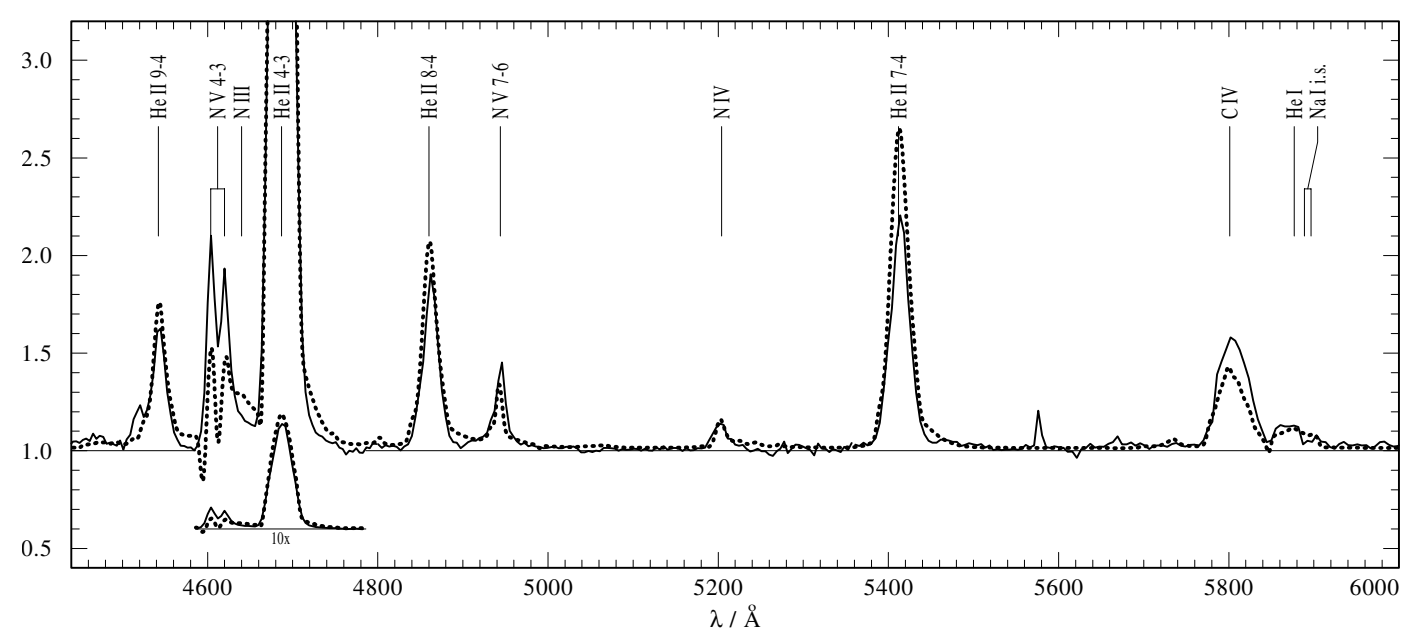

Fig. 2. Observed spectrum of WR 44 (WN4-w, continuous line), compared with model 09-12 $\left(T_{*}=63.1 \mathrm{kK}, \log R_{\mathrm{t}} / R_{\odot}=0.9\right)$ from the WNE grid. The synthetic spectrum is a good fit fit to the observed He II lines and He I 5876. The same model reproduces N v $4933-4944$ and N v 4603,4619, although the latter is a bit too weak. The unblanketed models used in Paper I were not able to reproduce He I and N v lines simultaneously.

spectrum. Thus the present best-fit model (09-12) differs only by one step in each of the grid parameters from the result adopted in Paper I for this particular star. As we will show in a forthcoming paper, for some other stars the revision of the parameters is more dramatic. In any case, the more consistent fit now provides a much safer ground for a quantitative spectral analysis.

For orientation in the parameter space, we visualize the model grids in the form of contour plots for the equivalent widths of some strategic emission lines. For this purpose we have re-calculated the formal integral for the considered individual lines or multiplets, omitting all blends and switching off the electron-scattering frequency redistribution.

Figure 3 shows contours of constant emission line equivalent width for three classification lines. The reader may again refer to the corresponding figures in Paper I in order to see the impact of the iron line blanketing. The "mountain" of the strong N III multiplet at 4634-4641 А (top panel) reaches its maximum at stellar temperatures $T_{*}$ of $60 \mathrm{kK}$ and more; compared to the unblanketed models of Paper I, it has shifted to considerably higher temperatures. The N IV line at $4058 \AA$ becomes strong at only slightly higher temperatures. The N v 4933-4944 blend is pronounced at $T_{*}$ higher than about $80 \mathrm{kK}$ if $R_{\mathrm{t}}$ is in the suitable range. Note that the $\mathrm{N}$ V maximum is also stretched towards the lower-right corner of the diagram, similar to all other contour "mountains". In contrast, the N V contours in Paper I looked like an isolated maximum, which now turned out to be wrong because of numerical deficiencies of the temperature structure in the old model calculations.

The contour plots for He II 5412 and He I 5876 are shown in Fig. 4. Compared to the unblanketed models, the He I contours are shifted a bit towards higher temperatures.

The classification scheme for WN stars can be applied to the model spectra in order to establish the domains of the different subtypes (Fig. 5). From the compilation by van der Hucht (2001) we adopt the following criteria. WN8: N III $4640 \simeq$ He II 4686; the corresponding thick grey line in Fig. 5 shows the contour where $W_{\lambda}\left(\mathrm{N}\right.$ III 4640) $=0.5 \times W_{\lambda}(\mathrm{He}$ II 4686). WN6: $\mathrm{N}$ III $4640 \simeq \mathrm{N}$ IV 4058; the corresponding thick grey line shows the contour where the equivalent widths are equal. WN4: N IV $4058 \simeq$ N v 4933-4944; shown is the contour where both $W_{\lambda}$ are equal. WN2.5: N IV 4058 absent; taking $1 \AA$ as detection limit, this domain lies to the right of the contour shown in the figure. The domain of early-type WN stars with strong lines (WNE-s) is enclosed by the dashed contour for $W_{\lambda}($ He II 5412) > $37 \AA$. As stated by Smith et al. (1996), this line-strength criterion is essentially equivalent to the alternative broad-line classification WNEb.

\section{The parameter degeneracy for very thick winds}

From the diagrams shown in the previous sections one can see that at small transformed radius all contours become more or less parallel lines. Their slope approaches $R_{\mathrm{t}} \propto T_{*}^{-2}$, corresponding to a constant mass-loss rate. In Fig. 6 we compare spectra of two grid models lying along such a line of same mass-loss rate, namely the models $11-17\left(T_{*}=79.4 \mathrm{kK}\right.$, $\left.\log R_{\mathrm{t}} / R_{\odot}=0.4\right)$ and $15-21\left(T_{*}=126 \mathrm{kK}, \log R_{\mathrm{t}} / R_{\odot}=0\right)$. Despite the different parameters, the two spectra are very similar. This holds not only for the normalized line spectra, but also for the absolute fluxes of the spectral energy distribution. As a consequence, when one analyzes an observed spectrum which happens to lie in this parameter range, $T_{*}$ and $R_{\mathrm{t}}$ cannot be uniquely determined. In Hamann et al. (2003) we therefore proposed to use $T_{*}=100 \mathrm{kK}$ as reference and quote the transformed radius $R_{\mathrm{t} 100 \mathrm{kK}}$ for such parameter-degenerate models or stars.

To understand this further degeneracy in the parameter space of WR atmospheres, imagine a very dense stellar wind. The terminal speed is already reached before it becomes optically thin at any frequency. Then (for given $v_{\infty}$, composition etc.) the object is fully described by its luminosity and massloss rate. To quote an effective temperature one may define any arbitrary reference radius, e.g. of a hydrostatic core, but this has 


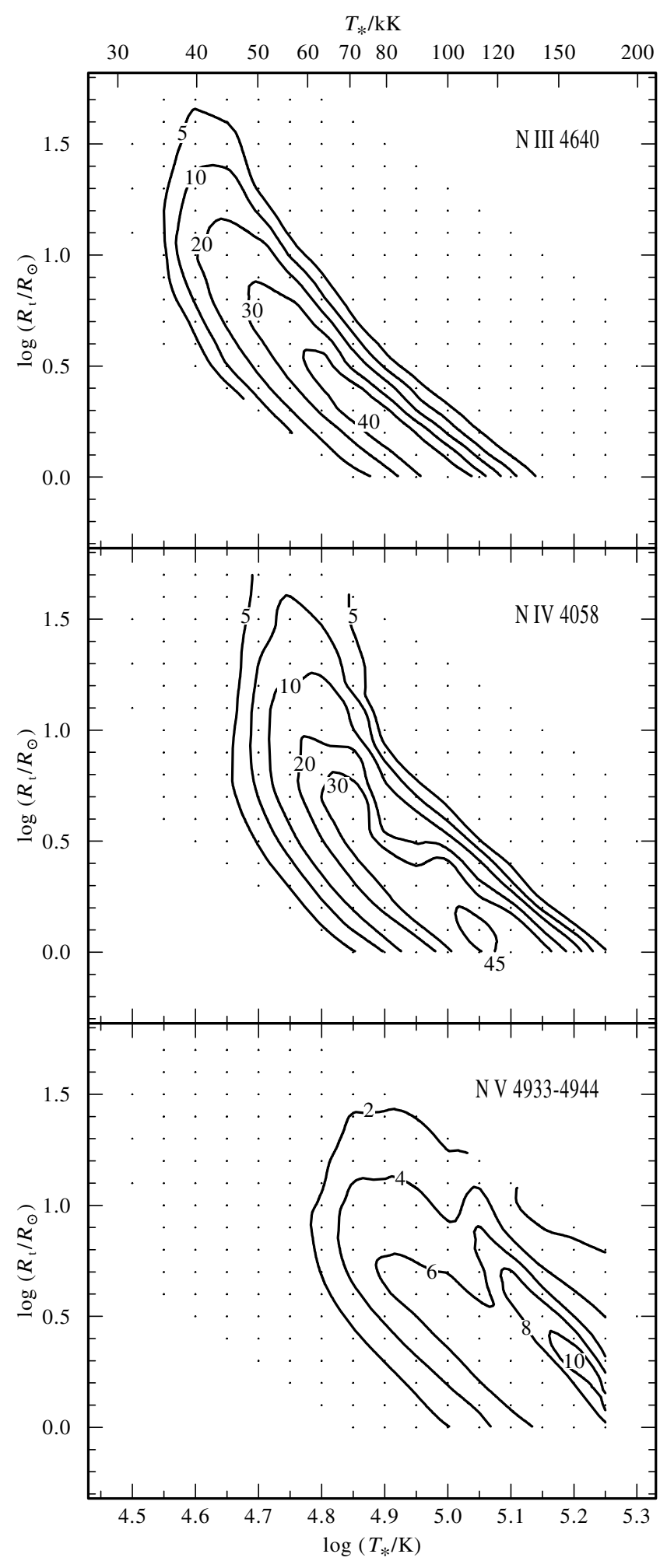

Fig. 3. Contours of constant emission line equivalent width (labels: $\AA$ ) for WN classification lines of nitrogen. Tiny dots indicate the calculated grid models (WNE grid).

no relevance for the emerging radiation, which comes from an optical depth around unity. The corresponding radius is basically a function of the mass-loss rate. Let us define $R_{2 / 3}$ as the radius where the radial Rosseland optical depth reaches $2 / 3$. For a given luminosity $L$ the effective temperature related to

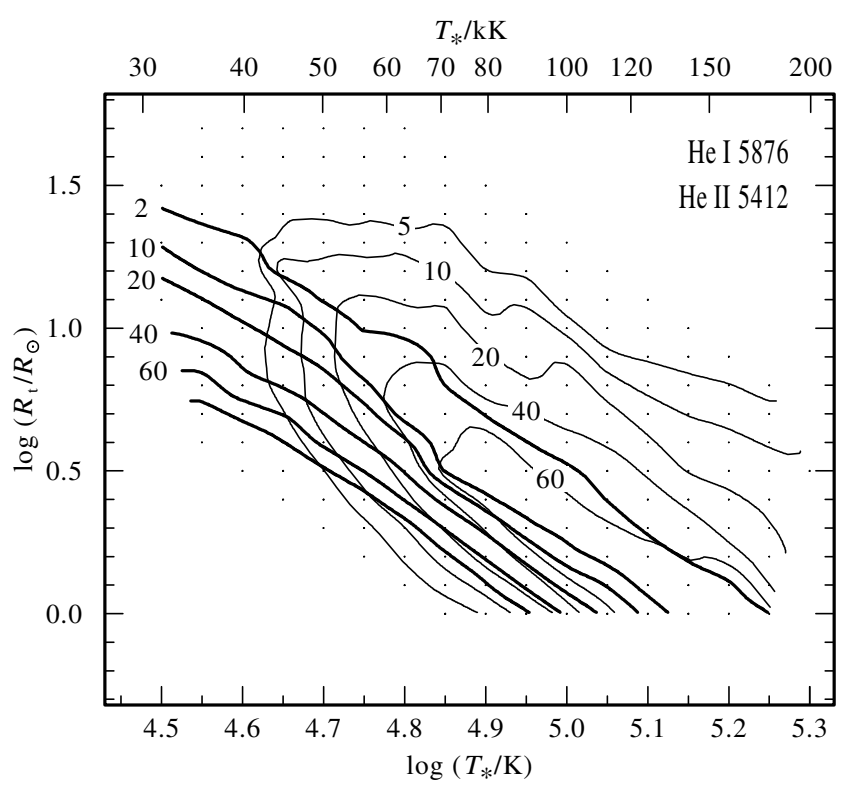

Fig. 4. Contours of constant line emission, labelled with the equivalent width in $\AA$, for strategic helium lines (thick contours: He I, thin: He II). Tiny dots indicate the calculated grid models (WNE grid).

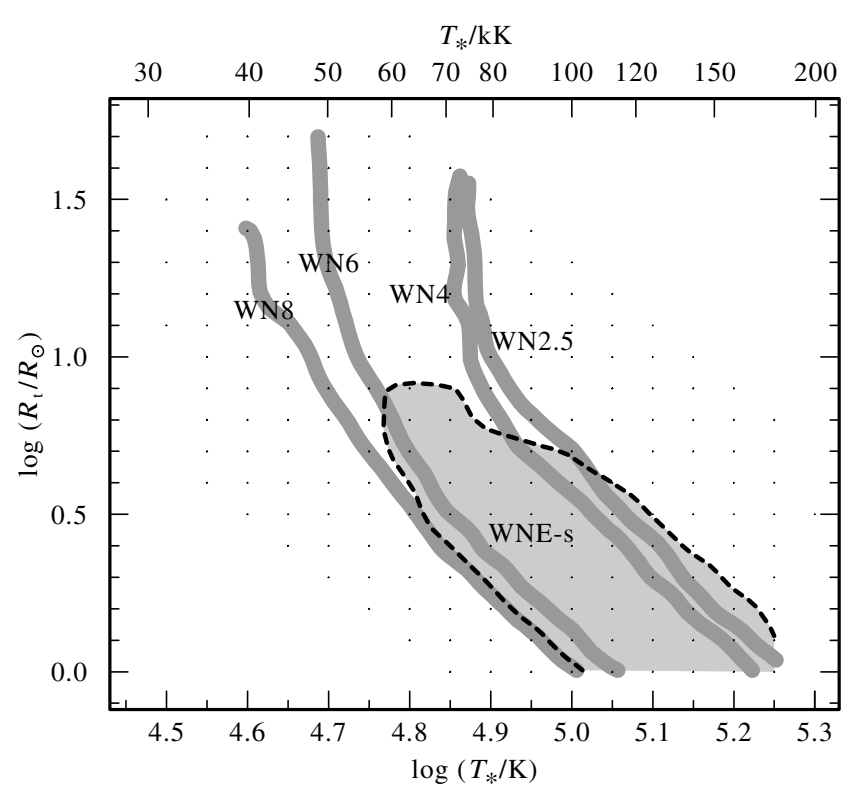

Fig. 5. Subtype classification (cf. Smith 1968; van der Hucht 2001) of the synthetic spectra in the WNE grid. Thick grey lines indicate where the emission line equivalent widths fulfill the following criteria. WN8: N III $4640=0.5 \times$ He II 4686; WN6: N III $4640=$ N IV 4058; WN4: N IV 4058 = N v 4933-4944; WN2.5: N IV $4058=1$ А. The dashed contour encloses the region in which He II 5412 is stronger than $37 \AA$, leading to the classification as WNE-s.

that radius, $T_{2 / 3}$, follows from Stefan-Boltzmann's law. Hence $\dot{M}$ uniquely specifies a model of given $L$. Allowing for different luminosities and considering the approximate scaling property discussed in Sect. 3, the spectrum depends only on the ratio $L / \dot{M}^{4 / 3}$ apart from a scaling of the absolute fluxes $\propto L$.

A completely different situation is encountered in less dense atmospheres, where the observed spectrum emerges from layers where the expansion is still small. Then the radius 


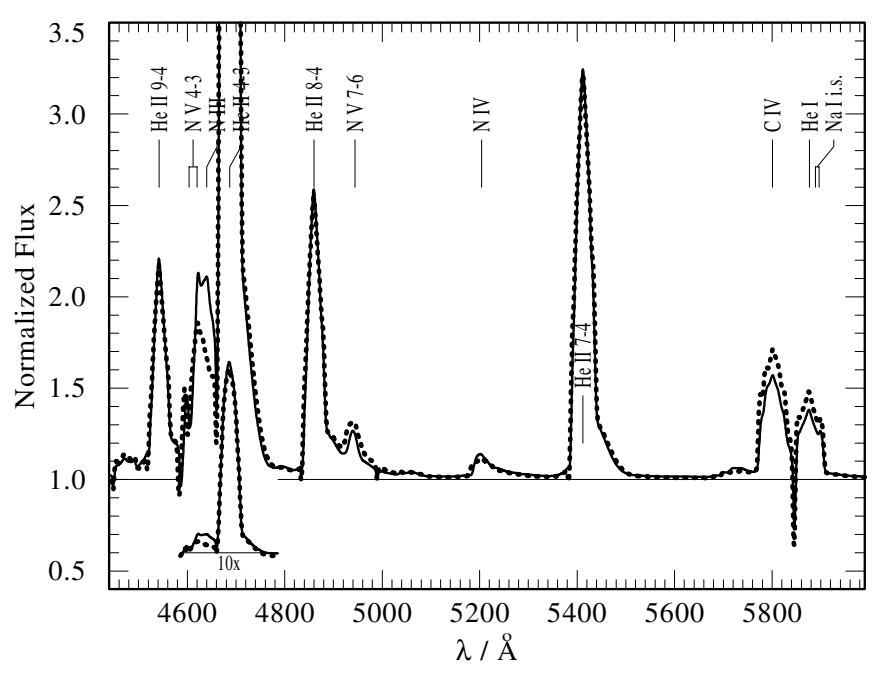

Fig. 6. Spectra of two grid models with equal mass-loss rate, 11-17 (solid line; $T_{*}=79.4 \mathrm{kK}, \log R_{\mathrm{t}}=0.4$ ) and 15-21 (dotted; $T_{*}=126 \mathrm{kK}, \log R_{\mathrm{t}}=0$ ). Despite the different parameters, the two spectra are very similar due to a parameter degeneracy of very dense Wolf-Rayet atmospheres.

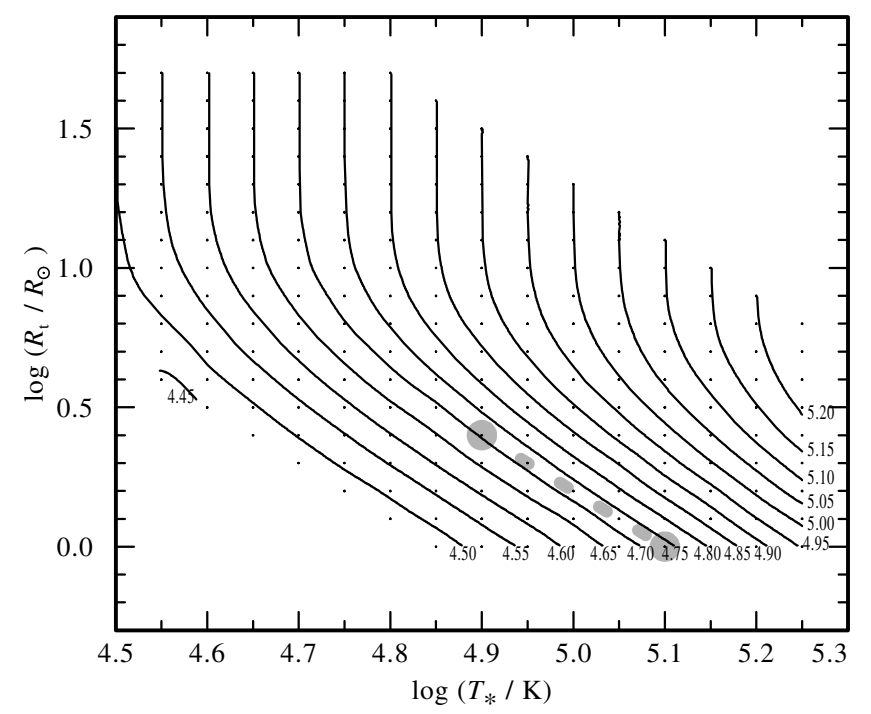

Fig. 7. Contours of equal temperature $T_{2 / 3}$ (labels: $\log T_{2 / 3} / \mathrm{K}$ ), defined via Stefan-Boltzmann's law as the effective temperature referring to the radius where the radial Rosseland optical depth reaches $2 / 3$. For models with low wind density (upper half of the diagram) $T_{2 / 3}$ is almost equal to $T_{*}$, i.e. to the effective temperature parameter of our models which refers to almost static layers (Rosseland optical depth of 20). For the more dense models (lower half of the diagram) the effective temperature at $\tau_{\text {Ross }}=2 / 3$ becomes independent of $T_{*}$ and is constant for models of equal mass-loss rate. The two models of equal mass-loss rate for which the spectra are compared in Fig. 6 are marked.

of that layer, which we term $R_{*}$, or the corresponding effective temperature $T_{*}$, is a further free parameter of the model.

For illustration Fig. 7 shows contours of equal $T_{2 / 3}=$ $T_{*}\left(R_{*} / R_{2 / 3}\right)^{1 / 2}$ for our WNE grid. For thin winds (large $R_{\mathrm{t}}$ ) $T_{2 / 3} \simeq T_{*}$ holds, while for thick winds the contours approach lines of constant mass-loss rate. WNE stars with strong lines (WNE-s) typically fall into the parameter region where this degeneracy starts to be effective.

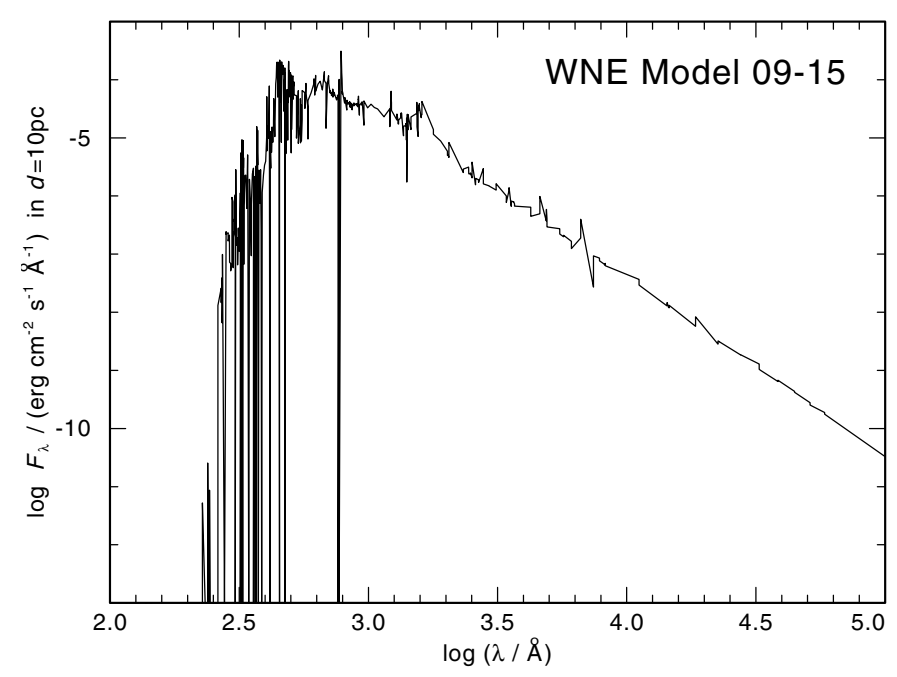

Fig. 8. Spectral energy distribution: preview plot as obtained from the PoWR web interface. The SED is shown on a coarse grid of frequency points which does not resolve individual line profiles, but gives correct frequency integrals.

\section{The web interface}

The synthetic spectra of the WN models discussed in the present paper are fully accessible via the World Wide Web. The Potsdam Wolf-Rayet (PoWR) homepage ${ }^{1}$ offers the choice between different grids of models; so far, the two WN grids presented in this paper ("WNE": hydrogen-free models; "WNL": models with $20 \%$ hydrogen) are available. Additional model grids will be added to the database in future.

After a model grid has been chosen, a specific model can be selected by mouse-click, which means defining the stellar temperature $T_{*}$ and the transformed radius $R_{\mathrm{t}}$. The synthetic spectrum is then offered in different forms. The spectral energy distribution (SED) is the entire flux spectrum, as seen by an observer at $10 \mathrm{pc}$ distance from the star. The SED is given on a coarse grid of frequency points which does not resolve individual line profiles (cf. Fig. 8). Despite the coarse frequency spacing, correct frequency integrals are always retained.

An extra button ("Colors") provides a list of photometric quantities of the model, such as the absolute monochromatic magnitudes $u, b, v$ (defined by Smith 1968). Moreover, the number of ionizing photons for $\mathrm{H}, \mathrm{He}$ and $\mathrm{He}^{+}$are given in that table. We compared these numbers with the WN model series published by Smith et al. (2002). For $T_{*} \geq 60 \mathrm{kK}$ we find perfect agreement between their ionizing photon fluxes (called $Q_{0}, Q_{1}$ and $Q_{2}$ therein) and our correspondingly interpolated grid model results, but for cooler stellar temperatures our models show significantly stronger line blanketing in the He I continuum. A warning should be issued concerning the He II continuum $(\lambda<228 \AA)$. In the cooler and denser models of the grids, this continuum is still optically thick at the outer boundary of the model atmosphere (at $1000 R_{\odot}$ ). Hence the calculation cannot provide meaningful emergent fluxes in those cases, as reflected by a 0.00 entry in our list of "Colors".

\footnotetext{
${ }^{1}$ http://www.astro.physik. uni-potsdam.de/PoWR.html
} 


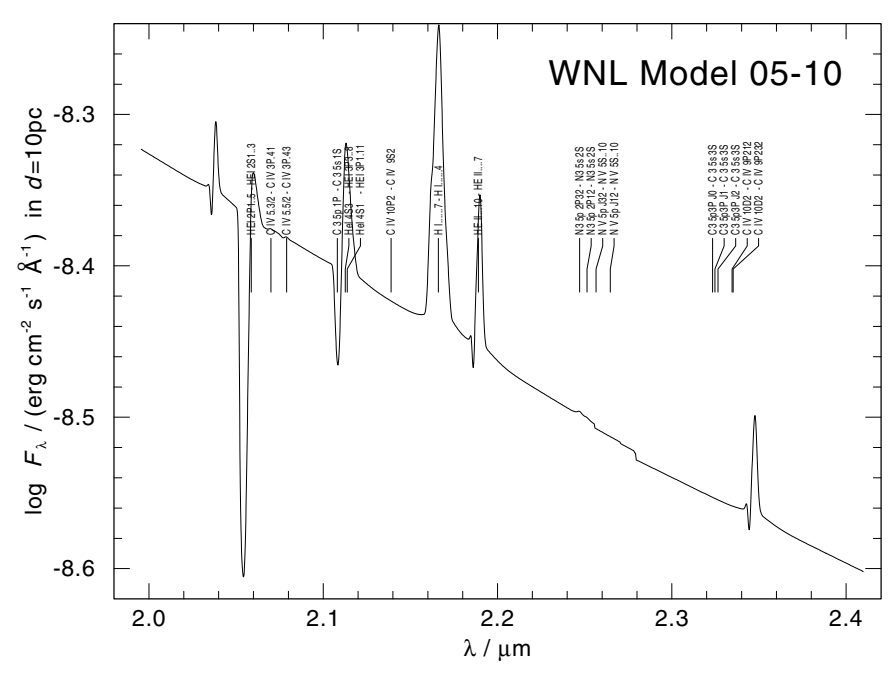

Fig. 9. Line spectrum: preview plot as obtained from the PoWR web interface. In this example, the IR $K$-band range was selected. Instead of the calibrated spectrum one can alternatively chose the normalized spectrum. Line identifications are optionally shown for all lines which are accounted for in the formal integral, provided that the oscillator strength $f$ is greater than 0.05 .

The line spectra are available as calibrated spectra (in absolute flux units, cf. Fig. 9), and as normalized spectra in units of the continuum. The spectral resolution corresponds roughly to $0.3 v_{\text {D }}$, i.e. $R \approx 10^{4}$. Optionally, lines which are accounted for in the formal integral are labeled in the preview plots. However, to avoid crowding, the line identifications are suppressed for lines with an oscillator strength smaller than $f=0.05$. The line spectra are organized in certain passbands (such as UV, visual spectrum, $K$-Band, IR 2.4-5.2 $\mu$ ), but sub-ranges can be selected. The selected data can be previewed with the web browser or as PostScript files, and the data can be downloaded in the form of AscII tables.

\section{Outlook}

The model grids presented here are ready for use for different applications. The line spectra can serve for spectral analyses, based on the various spectral bands from UV till $5 \mu$ in the infrared. Moreover, the spectral energy distributions might be useful for population synthesis or the extraction of ionizing fluxes.

Analyses of the Galactic WN stars with the new model grids are already in progress in our group and will be published soon, leading partly to substantial revisions of the stellar parameters. For an application to extragalactic systems, grids of different metallicities will be provided in the future.

Corresponding grids for WC stars are in preparation. For this class the abundances (He:C:O) provide one additional dimension in the parameter space. On the other hand, many of the WC stars fall into the regime of the parameter degeneracy for very dense winds described in Sect. 4.

Hydrodynamically consistent models for WR atmospheres are not yet available. For the models presented here the mass-loss rate is a free parameter, and the velocity law is assumed ad-hoc. However, hydrodynamically consistent
WR models are now successfully under construction in our group (Gräfener et al. in preparation). With the help of clumping, and of many more ionization stages of iron, radiation pressure can account for the acceleration of the dense Wolf-Rayet wind.

Acknowledgements. We acknowledge useful suggestions by the referee P. A. Crowther.

\section{References}

Conti, P. S., Leep, E. M., \& Perry, D. N. 1983, A\&A, 268, 228

Crowther, P. A. 2003, in A Massive Star Odyssey, from Main Sequence to Supernova, ed. K. A. van der Hucht, A. Herrero, \& C. Esteban, Proc. IAU Symp., 212, 47

Crowther, P. A., Fullerton, A. W., Hillier, D. J., et al. 2000, ApJ, 538, L51

Crowther, P. A., Dessart, L., Hillier, D. J., et al. 2002, A\&A, 392, 653

Dessart, L., Crowther, P. A., Hillier, D. J., et al. 2000, MNRAS, 315, 407

Gräfener, G., Hamann, W.-R., Hillier, D. J., \& Koesterke, L. 1998, A\&A, 329, 190

Gräfener, G., Koesterke, L., \& Hamann, W.-R. 2002, A\&A, 387, 244

Hamann, W.-R. 2003, in Stellar Atmosphere Modelling, ed. I. Hubeny, D. Mihalas, \& K. Werner, ASP Conf. Ser., 288, 171

Hamann, W.-R., \& Gräfener, G. 2003, A\&A, 410, 993

Hamann, W.-R., \& Koesterke, L. 1998a, A\&A, 333, 251 (Paper I)

Hamann, W.-R., \& Koesterke, L. 1998b, A\&A, 335, 1003

Hamann, W.-R., \& Koesterke, L. 2000, A\&A, 360, 647

Hamann, W.-R., Dünnebeil, G., Koesterke, L., Schmutz, W., \& Wessolowski, U. 1991, A\&A, 249, 443

Hamann, W.-R., Gräfener, G., \& Koesterke L. 2003, in A Massive Star Odyssey, from Main Sequence to Supernova, ed. K. A. van der Hucht, A. Herrero, \& C. Esteban, Proc. IAU Symp., 212, 198

Hamann, W.-R., Koesterke, L., \& Wessolowski, U. 1993, A\&A, 274, 397

Hamann, W.-R., \& Schmutz, W. 1987, A\&A, 174, 173

Hamann, W.-R., Koesterke, L., \& Wessolowski, U. 1995, A\&A, 299, 151

Hamann, W.-R., Wessolowski, U., \& Koesterke, L. 1994, A\&A, 281, 184

Herald, J. E., Hillier, D. J., \& Schulte-Ladbeck, R. E. 2001, ApJ, 548, 932

Hillier, D. J. 1987a, ApJS, 63, 947

Hillier, D. J. 1987b, ApJS, 63, 965

Hillier, D. J., \& Miller, D. L. 1998, ApJ, 496, 407

Hillier, D. J., \& Miller, D. L. 1999, ApJ, 519, 354

van der Hucht, K. A. 2001, New Astron. Rev., 45, 135

Koesterke, L., \& Hamann, W.-R. 1995, A\&A, 299, 503

Koesterke, L., Hamann, W.-R., \& Gräfener, G. 2002, A\&A, 384, 562

Kurucz, R. L. 1991, in Stellar Atmospheres - Beyond Classical Models, ed. L. Crivellari, I. Hubeny, \& D. G. Hummer, NATO ASI Ser., 341, 441

Mihalas, D., \& Hummer, D. G. 1973, ApJ, 179, 827

Schmutz, W., Hamann, W.-R., \& Wessolowski, U. 1989, A\&A, 210, 236

Smith, L. F. 1968, MNRAS, 140, 409

Smith, L. F., Shara, M. M., \& Moffat, A. F. J. 1996, MNRAS, 281, 163

Smith, L. J., Norris, R. P. F., \& Crowther, P. A. 2002, MNRAS, 337, 1309 